\title{
POSSIBLE EVOLUTIONARY INTERPRETATION OF THE DEPENDENCES ON THE DIAGRAMS FOR
}

\author{
RR LYRAE VARIABLES
}

\author{
V. P. GORANSKIJ \\ Moscow University, Moscow, U.S.S.R.
}

The separation of $\mathrm{RR}_{a b}$ variables into two sequences on the amplitude-period diagram was found by Belserene (1954) for globular cluster variables, and by Detre (1965) for field variables. It is significant that the sequence of short period variables is steeper on this diagram than that for stars of longer period. Szeidl (1965) pointed out that other characteristics of the light curves and photometric quantities such as the asymmetry of light curve $\varepsilon$, mean color index $B-V$, median magnitude $m_{\text {med }}$, maximum magnitude $m_{\max }$ are also separated into distinct sequences. Figures 1, 2 and 3 show these sequences on the colour-magnitude, colour-period, period-amplitude (or period-maximum magnitude, minimum magnitude) diagrams for $\mathrm{RR}_{a b}$ variables in the globular clusters M3, $\omega$ Cen and in the dwarf galaxy in Draco. On these figures $R R_{a b}$ variables of the long period sequence are marked by crosses. The colour-magnitude and colourperiod diagrams for M3 were obtained by Roberts and Sandage (1955); the periodmaximum magnitude, minimum magnitude diagram obtained by Szeidl (1965) is constructed for stars measured by Roberts and Sandage. To get the most accurate $V$-magnitudes and colour indices for variables in $\omega$ Cen the data of Dickens and Saunders (1965) and Geyer and Szeidl (1970) have been averaged. The diagrams for RR Lyr variables in the Draco system were obtained by Baade and Swope (1961). The variables which show the Blazhko effect were excluded.

One can see on the diagrams for RR Lyrae variables that the long period sequence stars which depart from the basic sequences have a systematically higher luminosity than ordinary stars. The $R_{c}$ variables of increased luminosity (crosses) also have systematically greater periods.

This complex of phenomena on the diagrams for RR Lyrae variables can be treated by means of calculations of the evolution of models with core helium burning (Iben and Rood, 1970) and pulsation theory (Christy, 1966).

Jben and Rood calculated the evolution of low-mass models $\left(0.6-0.7 \mathfrak{M}_{\odot}\right)$ with helium burning in a core and hydrogen burning in a shell. An example of the evolutionary tracks of such models is illustrated in Figure 4 . The parameters of the models are shown on the figure. ZAHB (zero age horizontal branch) is a mass sequence of the initial models with a core mass of $0.475 \mathfrak{M}_{\odot}$. The separation between two adjacent marks along each track corresponds to a time interval of $5 \times 10^{6} \mathrm{yr}$. According to the calculations the star evolves to the blue on the HR diagram during the most prolonged stage of its life on the horizontal branch. The surface temperature of the star increases during this stage and the radius decreases. The luminosity of the star remains approx- 

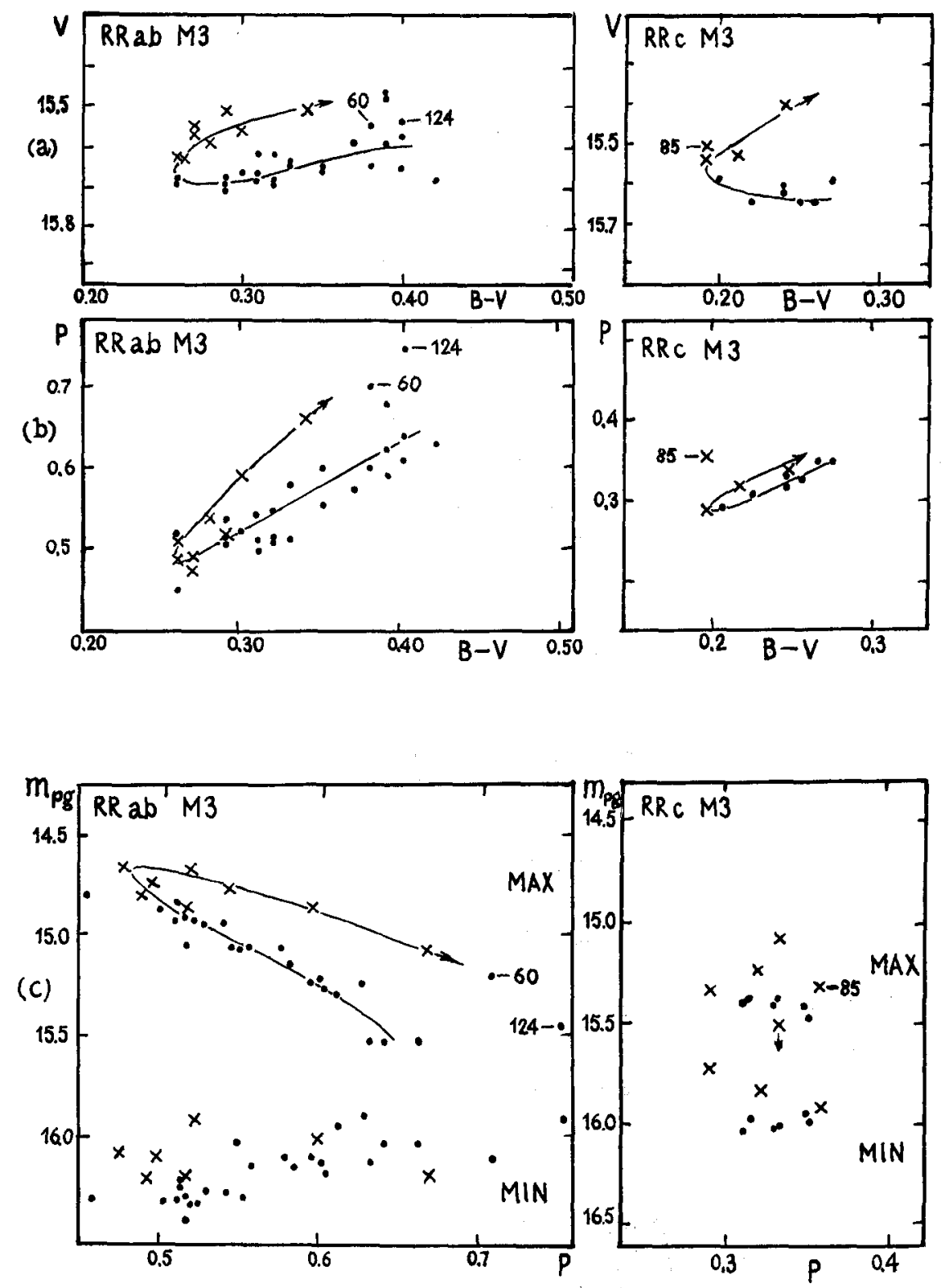

Fig. 1. The colour-magnitude (a), colour-period (b), period-maximum magnitude, minimum magnitude (c) diagrams for $\mathbf{R R}_{a b}$ and $\mathbf{R R}_{c}$ variables in the globular cluster M3. Solid lines are possible empirical tracks of variables.

imately constant. If pulsations are stimulated in the superficial layers of the star, the period of these pulsations will decrease.

On the $P-\log T_{e}$ diagram a variable will move approximately along the temperature sequence of models calculated by Christy for fixed mass, luminosity, and chemical 
composition (Figure 5). On the $A-P$ diagram a variable will shift along the sequence of models with the same fixed parameters (Figure 6). The group of variables of approximately equal mass at this stage of evolution will form the short period sequence.

According to the calculations, after relative exhaustion of the helium content in the core (to $30 \%$ of the initial content) the evolutionary changes undergo a revision. They lead to an increase of the luminosity and to an expansion of the envelope of the star. After the turn-off point of the evolutionary track the star evolves upwards and to the

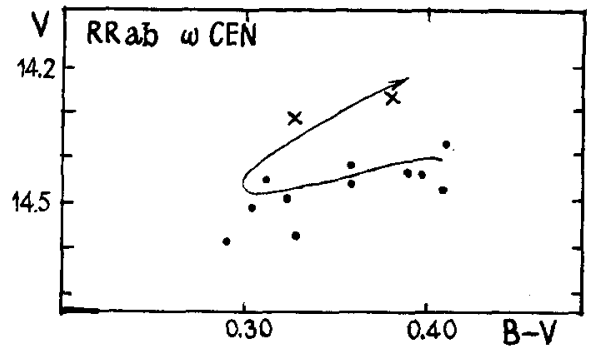

(a)

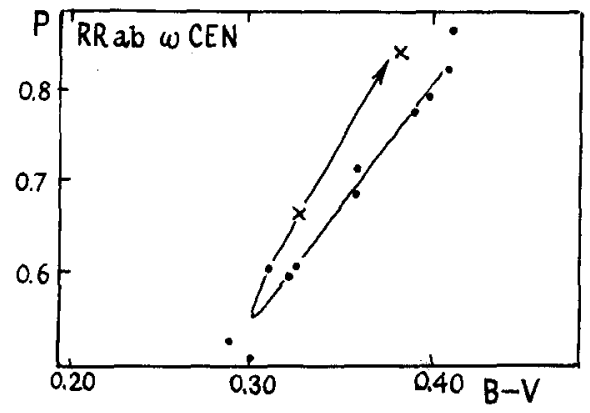

(b)
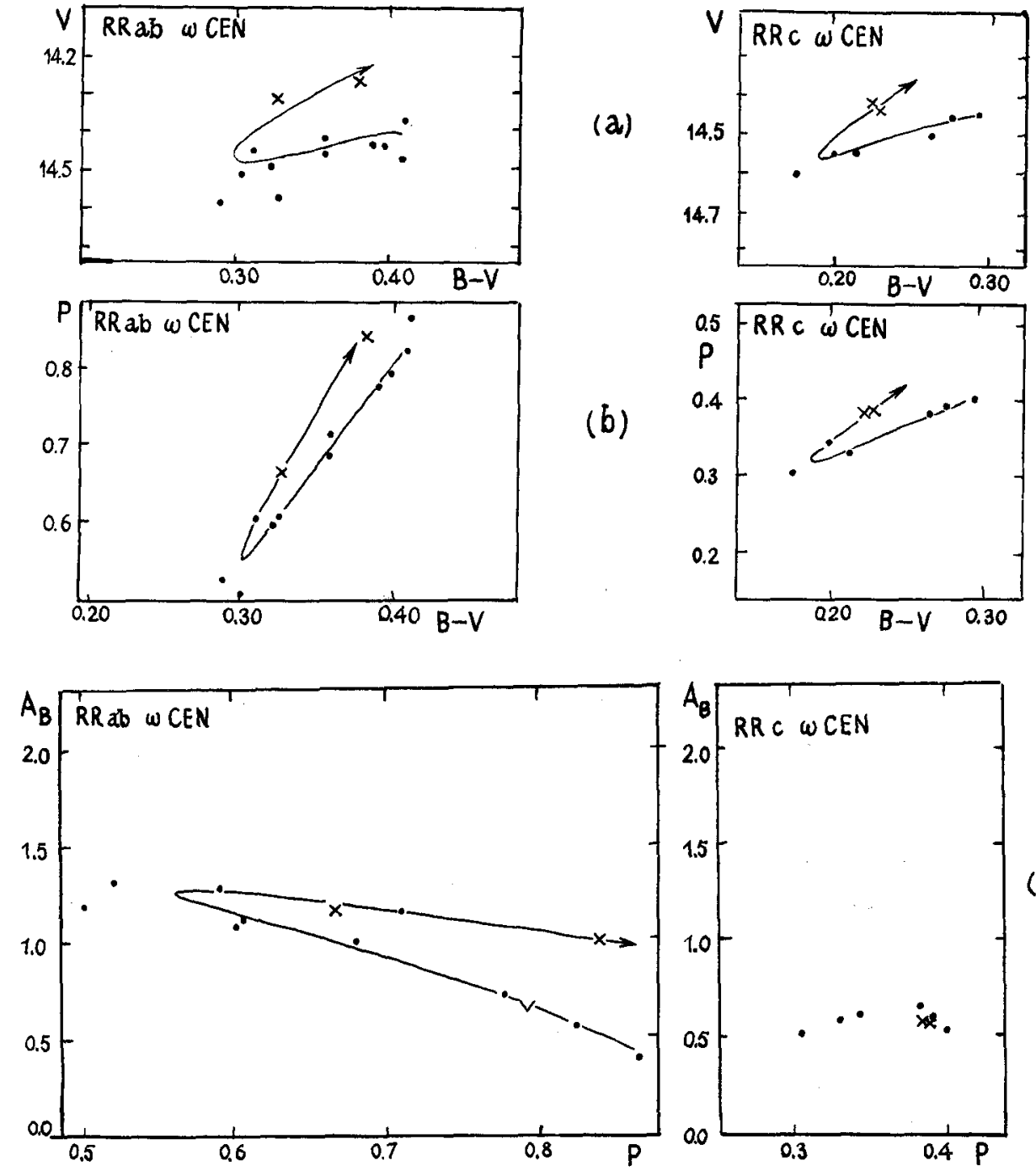

Fig. 2. The colour-magnitude (a), colour-period (b), period-amplitude (c) diagrams for $\mathbf{R}_{a b}$ and $\mathbf{R R}_{\boldsymbol{c}}$ variables in the globular cluster $\omega$ Cen. Solid lines are possible empirical tracks of variables. The stars known to show the Blazhko-effect are excluded. 

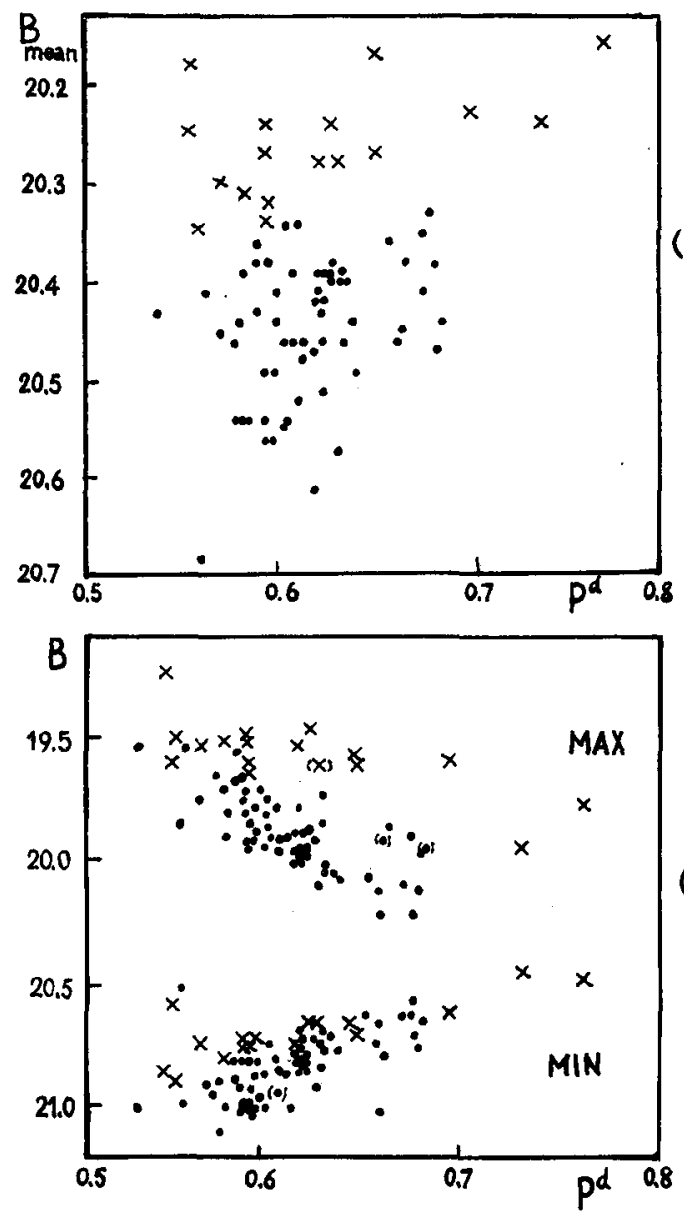

Fig. 3. The $B_{\text {mean }}-P$ (a) and $B_{\max }, B_{\min }-P(\mathrm{~b})$ diagrams for RR Lyrae variables in the Draco dwarf galaxy. Variables with the Blazhko-effect are excluded.

red on the HR diagram. The increase of the luminosity causes the deviation of the pulsating star upwards on the $P-\log T_{e}$ diagram (Figure 5). On the $A-P$ diagram the variables in such a phase of evolution deviate to the right, forming the long period sequence (Figure 6). Unfortunately all these stages of evolution can be illustrated on the theoretical diagrams for the RR Lyrae variables only schematically because Christy published sufficiently complete temperature sequences of the models with differing luminosities only for a helium content of $60 \%$.

The phenomena on similar diagrams for $R_{R_{c}}$ variables can also be treated by evolution theory. An unfilled wedgeshaped form of the $\mathrm{RR}_{a b}$ and $\mathrm{RR}_{c}$ variables on most diagrams suggest that these two groups have a very slight scattering of masses in each of them. Evidently the slight scattering of masses can also occur in the BHB stars (blue horizontal branch stars), as is seen in Figure 7, which shows the HR diagram for 


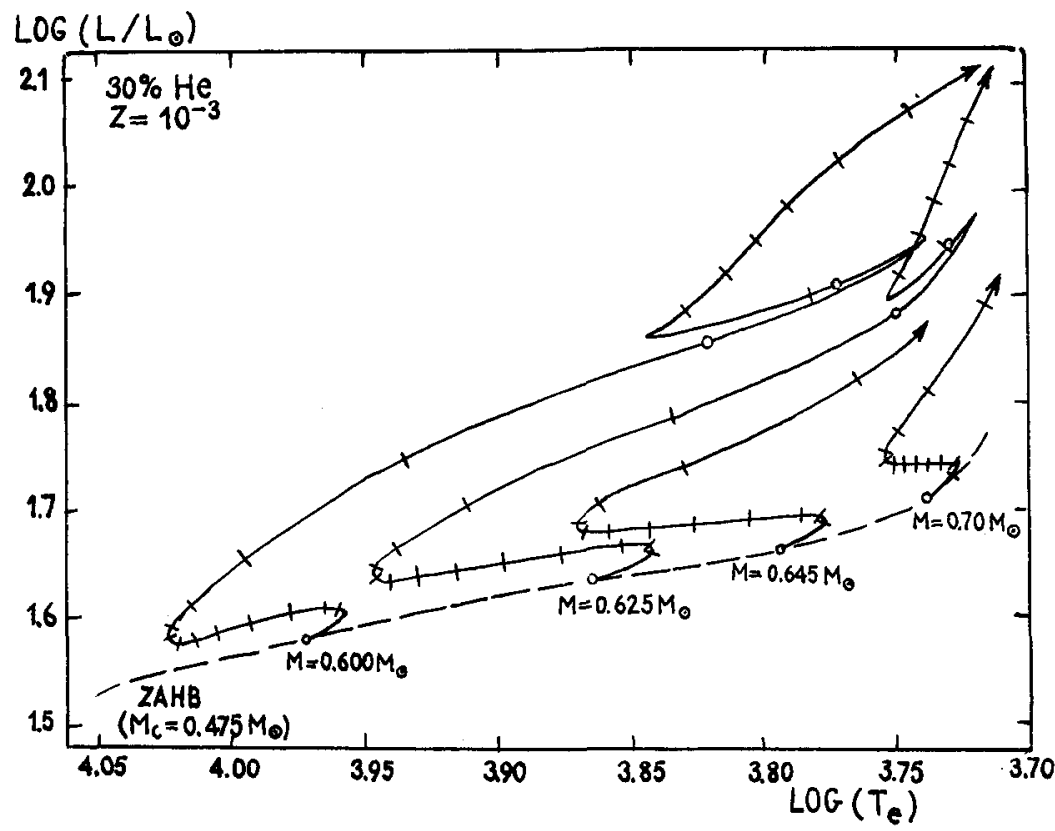

Fig. 4. Evolutionary tracks of low-mass models with helium burning in a core and hydrogen burning in a shell (Iben and Rood, 1970).

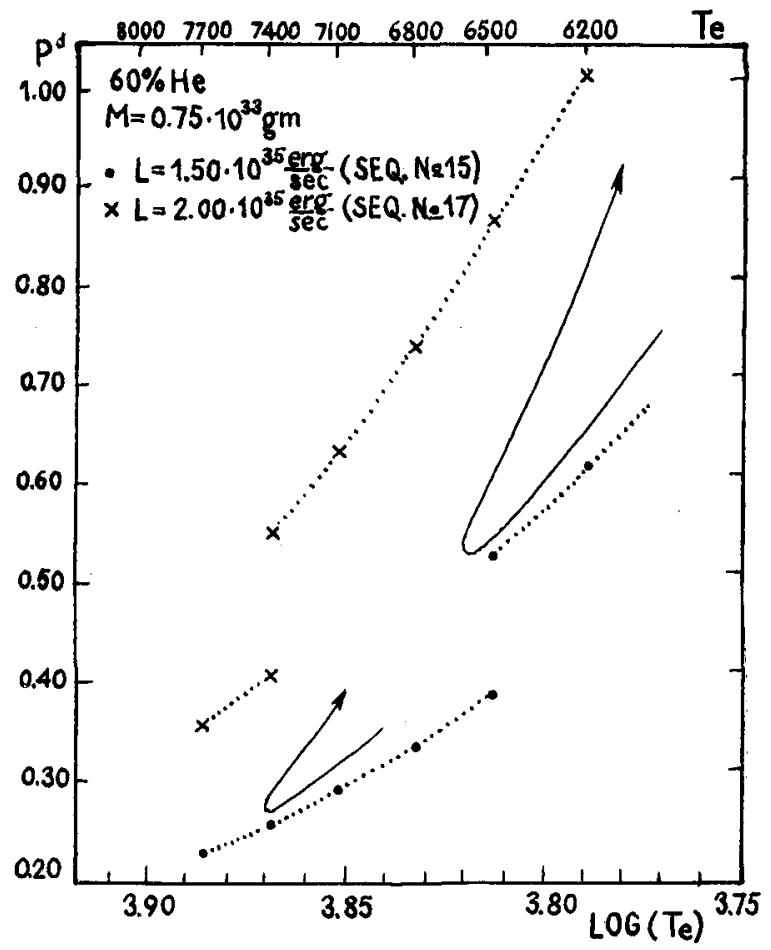

Fig. 5. Schematic representation of sequences in the period-colour diagram. Dotted lines are the temperature sequences of Christy. 


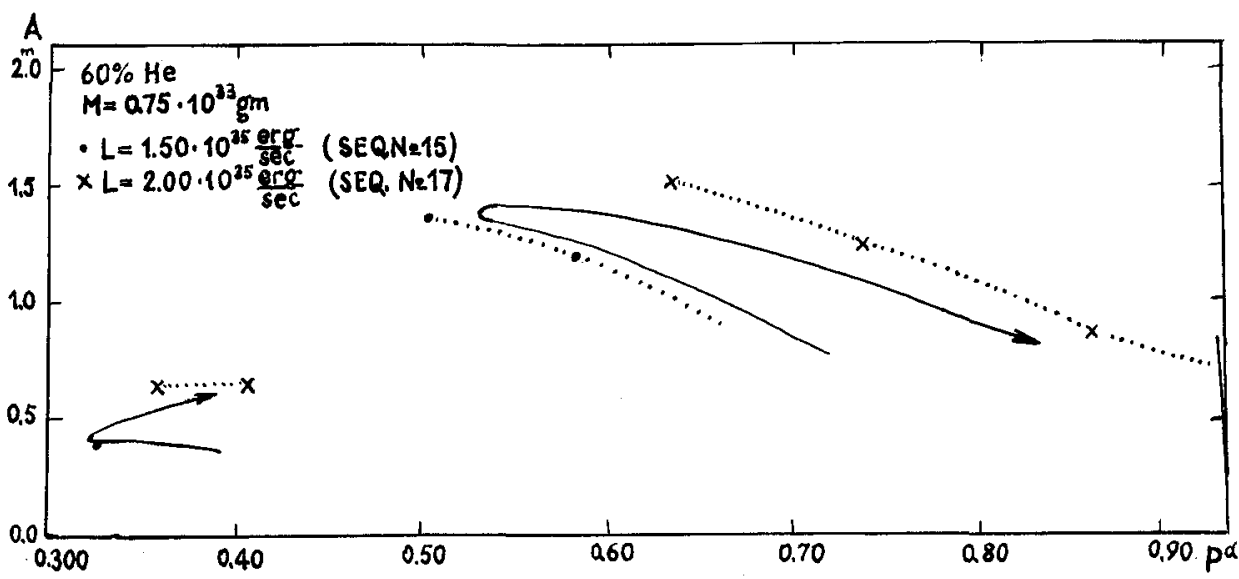

Fig. 6. Schematic representation of sequences in the period amplitude diagram. Dotted lines are the sequences of Christy.

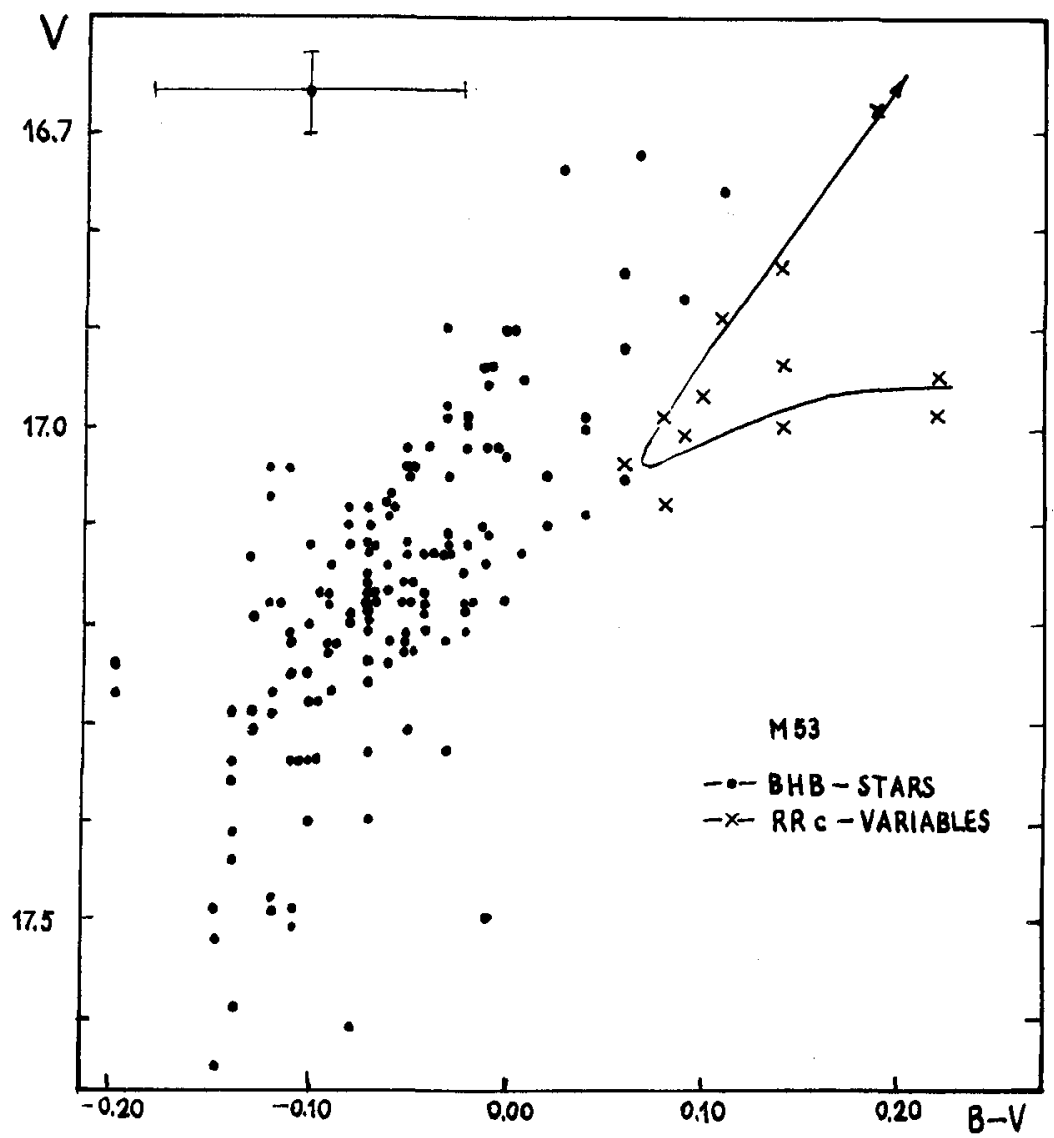

Fig. 7. The colour-magnitude diagram for $B H B$ stars and $\mathbf{R R}_{\boldsymbol{c}}$ variables in the globular cluster M53. The observed effect is of order of observational errors. 
BHB stars and $\mathrm{RR}_{c}$ variables in M53 (photometry of Cuffey, 1965). If the conjecture about the slight mass scattering in each group of variables is correct, then the solid lines in Figures 1 and 2 are the empirical tracks of variables on the diagrams. If the excluded variables with Blazhko-effect occur with equal frequency in these two stages of evolution, the number of variables in these stages is approximately in accordance with the rates of evolution calculated by Iben and Rood.

\section{References}

Baade, W. and Swope, H.: 1961, Astron. J. 66, 300.

Belserene, E. P.: 1954, Astron. J. 59, 406.

Christy, R. F.: 1966, Astrophys. J. 144, 108.

Cuffey, J.: 1965, Astron. J. 70, 732.

Detre, L.: 1965, in Transactions of the 4th Cosmogonical Conference, Moscow, p. 389.

Dickens, P. and Saunders, B.: 1970, Roy. Observ. Bull. No. 101

Geyer, E. and Szeidl, B.: 1970, Astron. Astrophys. 4, 40.

Iben, I. and Rood, R. T.: 1970, Astrophys. J. 161, 587.

Roberts, M. and Sandage, A.: 1955, Astron. J. 60, 185.

Szeidl, B.: 1965, Mitt. Sternw. Ungar. Acad. Wiss., Budapest-Szabadsaghegy No. 58.

\section{DISCUSSION}

Belserene: It fits in rather nicely with the period changes. The higher amplitude stars are the ones for which the observations are most apt to show increasing periods. 